\title{
Direct and semidirect radiative capture of nucleons with Hartree-Fock-BCS bound states
}

\author{
L. Bonneau ${ }^{1, a}$, T. Kawano ${ }^{1}$, T. Watanabe ${ }^{1,2}$, and S. Chiba ${ }^{3}$ \\ 1 Theoretical Division, Los Alamos National Laboratory, Los Alamos, New Mexico 87545, USA \\ 2 Department of Applied Quantum Physics and Nuclear Engineering, Kyushu University, 744 Motooka, Nishi-ku, Fukuoka 819-0395, Japan \\ 3 Advanced Science Research Center, Japan Atomic Energy Agency, Tokai, Ibaraki 319-1195, Japan
}

\begin{abstract}
Nucleon radiative capture is one of the most important process for nucleo-synthesis calculations in astrophysics. The nucleon capture can occur in two different mechanisms: the compound reaction and the directsemidirect (DSD) process. The compound capture cross sections become very small when many neutron channels open because the neutron width becomes much larger than the $\gamma$ width. For incident nucleon energies above about $5 \mathrm{MeV}$, the capture process can be described by the DSD theory only. In the DSD process, the incident particle is captured directly by an unoccupied bound state (direct) or it excites a collective state and is then scattered into a bound state (semidirect). In this picture, the calculation is sensitive to the radial wave functions of the bound state, which are often calculated with a single-particle model using a Wood-Saxon potential. For astrophysical calculations, since experimental information on nuclear structure is uncertain or inaccessible, we apply a Hartree-Fock-BCS (HFBCS) structure model to generate the radial wave functions. The DSD cross sections are obtained by calculating a transition amplitude to the HFBCS states and using the calculated spectroscopic factors. We calculate the neutron capture cross sections for even-even spherical and deformed targets, namely ${ }^{208} \mathrm{~Pb},{ }^{122,132} \mathrm{Sn}$ and ${ }^{238} \mathrm{U}$. The agreement with the experimental cross sections, only available for ${ }^{208} \mathrm{~Pb}$ and ${ }^{238} \mathrm{U}$, is very good.
\end{abstract}

\section{Introduction}

The nucleon radiative capture is one of the most important processes for nucleosynthesis calculations in Astrophysics. The incident-neutron energy range of interest depends on the temperature of the Maxwell-Boltzmann velocity distribution [1]. Neutron capture cross sections up to several $\mathrm{MeV}$ may meet astrophysical conditions in many cases. In this energy region, the statistical Hauser-Feshbach model with width fluctuation correction is known to be applicable [2] to calculate the astrophysical nuclear reaction rates, and extensive studies have been made (see ref. [3] for example).

In compound nuclear reactions, the capture cross sections become very small when neutron inelastic channels open, because the neutron width $\Gamma_{n}$ becomes much larger than the gamma-ray width $\Gamma_{\gamma}$. For incident nucleon energies above $5 \mathrm{MeV}$ or so, the capture process can be mainly described by the direct-semidirect (DSD) mechanism [4,5]. In this mechanism, the incident particle is captured directly in an unoccupied bound state (direct), or it excites a collective (giant dipole resonance) state and then is scattered into a bound state (semidirect). Within the perturbation theory, the transition probabilities of these processes involve radial wave functions and spectroscopic factors of the final states. The radial part of the wave function is often calculated with a single-particle model assuming that the potential has a spherical Wood-Saxon shape and the experimental spectroscopic factors $S$ are used if available.

The experimental information on nuclear structure for unstable nuclei being often uncertain or unavailable, we have to resort to model calculations to determine the DSD cross section. This is why we apply the Hartree-Fock-BCS (HFBCS)

${ }^{a}$ Presenting author, e-mail: bonneau@lanl.gov model to calculate the radial wave-functions of the singleparticle bound states of even-even target nuclei and their associated occupation probabilities from which we deduce the spectroscopic factors as in $[6,7]$. We choose here the Skyrme interaction in the mean-field channel in two different parameterizations and the traditionally associated pairing interactions. The main interest of considering the HFBCS approach to nuclear structure here is that we can describe the ground-state properties of spherical as well as deformed nuclei (with axial symmetry assumed) in a predictive manner.

\section{Theoretical framework}

\subsection{Direct-semidirect cross section}

The DSD model for nucleon capture initially proposed by Brown [4] and Clement, Lane and Rook [5] was later on extended to deformed nuclei by Boisson and Jang [8]. The procedure outlined by Boisson-Jang was then followed by several studies [9-11] to reproduce experimental capture cross sections for deformed nuclei.

Let us consider an incident nucleon with wave number $k_{n}$ and orbital and total angular momenta $L$ and $J$, respectively. We denote by $R_{L J}(r)$ the radial part of its distorted wave function calculated within the optical model. This nucleon is captured by an even-even target initially in its ground state (initial spin 0) and scattered into a bound state $|k\rangle$. With the assumption of axial symmetry for the deformed target, the single-particle $|k\rangle$ is an eigenstate of $J_{z}$, the projection on the symmetry axis of the angular momentum operator $\mathbf{J}^{2}$. The associated eigenvalue is denoted by $K$. The deformed single-particle state $|k\rangle$ is a superposition of eigenstates of 
the orbital and total angular momentum operators $\mathbf{L}^{2}$ and $\mathbf{J}^{2}$, respectively:

$$
|k\rangle=\sum_{l, j} C_{l j}^{(k)}|l j K\rangle .
$$

The corresponding DSD cross section takes the form of a sum of two coherent amplitudes [8,9]

$$
\begin{aligned}
\sigma^{(k)}(l j ; L J)= & \frac{8 \pi}{9} \frac{\mu}{\hbar^{2}}\left(\frac{k_{\gamma}}{k_{n}}\right)^{3}\left\langle I_{i} K_{i} j K_{f} \mid I_{f} K_{f}\right\rangle^{2} \times \\
& \left|T_{d}^{(k)}(l j ; L J)+T_{s}^{(k)}(l j ; L J)\right|^{2},
\end{aligned}
$$

where $T_{d}^{(k)}$ and $T_{s}^{(k)}$ denote the direct and semidirect amplitudes, respectively, $\mu$ is the reduced mass of the projectiletarget system and $I_{i}$ is the angular momentum of the target with projection $K_{i}$ on its intrinsic symmetry axis $z$. The direct contribution is given by

$$
T_{d}^{(k)}(l j ; L J)=\bar{e}(-i)^{l+1} Z\left(L J l j ; \frac{1}{2} 1\right) \sqrt{S_{l j}^{(k)}}\left\langle\mathcal{R}_{l j}^{(k)}|r| R_{L J}\right\rangle,
$$

where $\bar{e}$ is the effective charge $(\bar{e}=N e / A$ for proton and $\bar{e}=-Z e / A$ for neutron), $S_{l j}^{(k)}$ is the spectroscopic factor and $Z\left(L J l j ; \frac{1}{2} 1\right)$ is the $Z$-coefficient [12]. As for the semidirectcapture amplitude, it is calculated as

$$
\begin{aligned}
& T_{s}^{(k)}(l j ; L J)= \pm \frac{3}{2\left\langle r^{2}\right\rangle} \frac{N^{2} Z^{2}}{A^{3}} e \times \\
& \sum_{l^{\prime} j^{\prime}}\left[(-i)^{l^{\prime}+1} Z\left(L J l^{\prime} j^{\prime} ; \frac{1}{2} 1\right) \sqrt{S_{l^{\prime} j^{\prime}}^{(k)}}\left\langle\mathcal{R}_{l^{\prime} j^{\prime}}^{(k)}|h(r)| R_{L J}\right\rangle \times\right. \\
& \sum_{v}\left\langle 1,-v, J, v+K \mid j^{\prime} K\right\rangle\langle 1,-v, J, v+K \mid j K\rangle \times \\
& \left.\frac{\left|\left\langle\psi_{1 v}\left|\rho_{\nu}^{\prime}\right| \psi_{00}\right\rangle\right|^{2}}{E_{n}-\left(E_{v}+\epsilon_{k}\right)+i \frac{1}{2} \Gamma_{v}}\right],
\end{aligned}
$$

where $\left\langle r^{2}\right\rangle$ is the mean square radius, $h(r)$ is the particlevibration coupling function, $\epsilon_{k}$ is the single-particle energy of the considered bound state, $E_{v}$ and $\Gamma_{v}$ are the giant dipole resonance energy and width, and the index $v$ stands for the modes corresponding to major and minor axes. When $h(r)$ is a surface coupling function [8], the factor $3 /\left\langle r^{2}\right\rangle$ must be omitted [13]. The transition matrix element between the dipole and ground states $\left\langle\psi_{1 v}\left|\rho_{v}^{\prime}\right| \psi_{00}\right\rangle$ can be related to a photoabsorption cross section [8,9]. The GDR parameters are taken from experimental data, or calculated with a simple systematics [14] obtained from the experimental database. Upon summing over all the bound states and their $(l, j)$ components, we finally obtain the total DSD cross section $\sigma_{\text {DSD }}$. In this work we adopt the same global optical potential of Koning and Delaroche [15] in the different mass regions considered to calculate the radial wave function of the scattering states. As for the particle-vibration coupling function $h(r)$ in equation (4), to which the semidirect capture cross section is sensitive, we employ here the complex function of the form $h(r)=V_{1} r f(r)-i W_{1} 4 a r d f(r) / d r$ proposed by Potokar [16] with the values $V_{1}=75$ and $W_{1}=140 \mathrm{MeV}$ for all considered nuclei obtained from the analysis of the ${ }^{208} \mathrm{~Pb}(n, \gamma)$ reaction.

\subsection{Radial wave-function and spectroscopic factors within the Hartree-Fock-BCS approach}

We consider here two different parameterizations of the Skyrme interaction in the mean-field channel with the traditionally associated pairing interactions. On the one hand, with the SLy4 parameterization [17], we use the densitydependent delta interaction (DDDI) of Duguet et al. [18] as a pairing interaction. On the other hand, the seniority force (constant matrix elements between pairs of time reversed states) is chosen as a pairing interaction while using the Skyrme SIII [19] parameterization in the mean-field channel. The strengths of the seniority force for neutrons and protons retained here are the same as those used in ref. [20]

Within the Hartree-Fock-BCS approach, the ground state $|\Psi\rangle$ of an even-even nucleus takes the form $|\Psi\rangle=\prod_{k>0}\left(u_{k}+\right.$ $\left.v_{k} a_{k}^{\dagger} a_{\bar{k}}^{\dagger}\right)|0\rangle$, where $|\bar{k}\rangle$ is the time-reversed conjugate of $|k\rangle$ and $a_{k}^{\dagger}$ is the single-particle state creation operator. The BCS variational parameters $u_{k}$ and $v_{k}$ are related through $u_{k}^{2}+v_{k}^{2}=1, v_{k}^{2}$ representing the occupation probability of $|k\rangle$, and this relation ensures that $|\Psi\rangle$ is normalized to unity. The single-particle wave function associated with $|k\rangle$ is solution to the HartreeFock equation [21,22], which is of the form of eigenvalue equation for the Hartree-Fock Hamiltonian. In practice, the Hartree-Fock equation is solved by iterative diagonalization. Owing to the axial symmetry assumed here, we perform this diagonalization in the cylindrical harmonic-oscillator basis and then expands it onto the spherical harmonic-oscillator basis, which yields $|k\rangle=\sum_{n, l, j} s_{n l j}^{(k)}|n l j K\rangle$. The coefficient $C_{l j}^{(k)}$ in equation (1) is therefore related to $s_{n l j}^{(k)}$ through

$$
\left|C_{l j}^{(k)}\right|^{2}=\sum_{n \geqslant 0}\left|s_{n l j}^{(k)}\right|^{2} .
$$

Finally the spectroscopic factor $S_{i j}^{(k)}$ for a capture reaction on an even-even target is given by

$$
S_{l j}^{(k)}=\frac{2 u_{k}^{2}}{2 j+1}\left|C_{l j}^{(k)}\right|^{2} .
$$

\section{Results and discussion}

\subsection{Occupation probabilities}

To avoid any confusion in the definition of the spectroscopic factor, we compare the calculated occupation probabilities $v_{k}^{2}$ for various bound states with the values extracted from experimental data for five even isotopes of tin from ${ }^{116} \mathrm{Sn}$ to ${ }^{124} \mathrm{Sn}$. These nuclei being found spherical, there is no ambiguity in the assignment of the quantum numbers $l$ and $j$ to the singleparticle states $|k\rangle$. The results obtained with the SLy4 Skyrme interaction and the DDDI pairing interaction, and with the SIII parameterization and the seniority pairing force, are displayed in table 1 . The overall agreement is fairly good within both HFBCS models. 
Table 1. Occupation probabilities of five neutron single-particle levels in the $N=50-82$ shell for even Sn isotopes within the $\mathrm{HF}(\mathrm{SIII})+\mathrm{BCS}(\mathrm{G})$ (column labeled "SLy4") and HF(SLy4) + BCS (DDDI) (column labeled "SIII") approaches. Experimental data are taken from ref. [7].

\begin{tabular}{cccc}
\hline \hline Level & SIII & SLy4 & exp. \\
\hline$h_{11 / 2}$ & 0.44 & 0.35 & 0.47 \\
$d_{3 / 2}$ & 0.54 & 0.74 & 0.59 \\
$s_{1 / 2}$ & 0.64 & 0.85 & 0.69 \\
$g_{7 / 2}$ & 0.96 & 0.92 & 0.92 \\
$d_{5 / 2}$ & 0.96 & 0.96 & 0.86 \\
\hline \hline
\end{tabular}

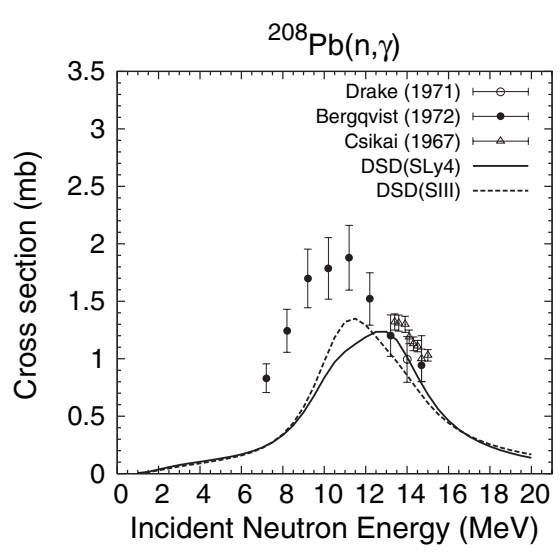

Fig. 1. Direct-semidirect neutron capture cross section for ${ }^{208} \mathrm{~Pb}$ with bound states calculated within two different HFBCS models.

\subsection{Capture cross sections}

First we calculate the DSD cross section with HFBCS bound states for the target nucleus ${ }^{208} \mathrm{~Pb}$. We use the GDR parameters $E_{0}=13.6 \mathrm{MeV}, \Gamma_{0}=3.78 \mathrm{MeV}$ and $\sigma_{0}=541 \mathrm{mb}$ taken from ref. [8]. The comparison of the calculated and experimental cross sections is shown in figure 1 . The calculated cross section has a shape consistent with the experimental data [2325], but the absolute cross section is slightly lower. A better reproduction of the data might be obtained with an appropriate adjustment of the $V_{1}$ and $W_{1}$ parameters of the Potokar particle-vibration coupling function. Two other causes to the underestimate of the cross section are the underbound character of the calculated bound states (due to particle-vibration coupling missing in the current Hartree-Fock description) and correlatively missing experimental bound states calculated to be quasi bound. A further investigation of these two effects has been carried out in ref. [26].

Then we perform capture cross section calculations for two tin isotopes, ${ }^{122} \mathrm{Sn}$ and ${ }^{132} \mathrm{Sn}$ (see fig. 2). The latter is of particular importance in the $r$-process of nucleosynthesis. Because of its doubly magic nature, ${ }^{132} \mathrm{Sn}$ has a very small compound radiative-capture cross section, and reaction rates for the ${ }^{132} \mathrm{Sn}(n, \gamma)$ and ${ }^{133} \mathrm{Sn}(\gamma, n)$ reactions become comparable in the r-process. As a result, relatively large amount of ${ }^{132} \mathrm{Sn}$ accumulates before it $\beta$-decays to ${ }^{132} \mathrm{Sb}$. In the upper panel of fig. 2 we have represented for ${ }^{122} \mathrm{Sn}$ the DSD cross sections with SLy4 and SIII as dashed and dash-dotted lines, respectively, and the corresponding compound-nucleus-plus-DSD
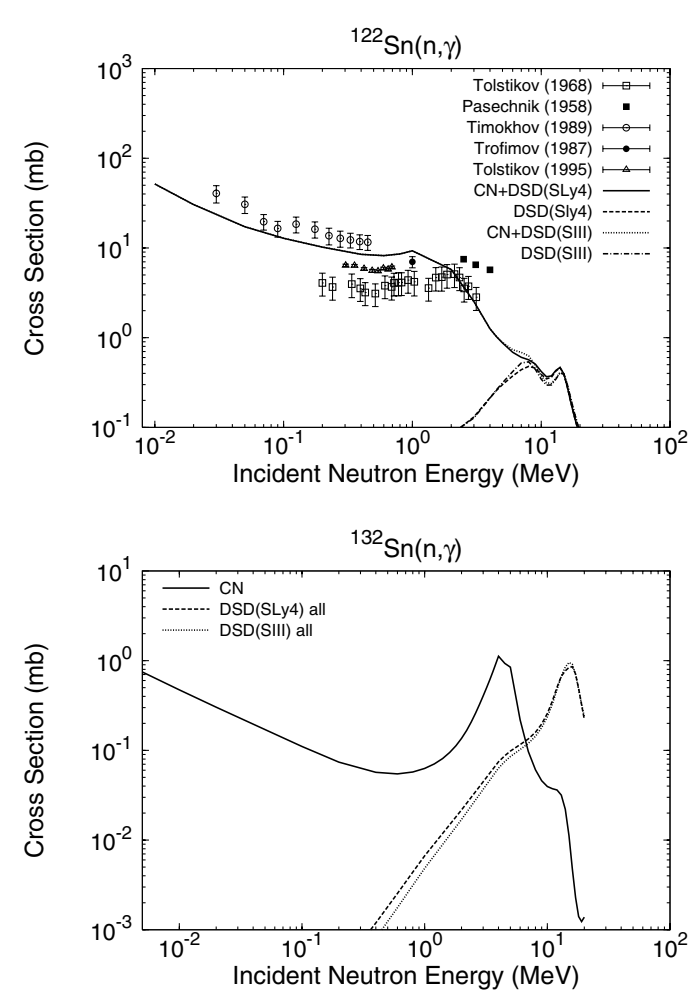

Fig. 2. Same as figure 1 for two tin isotopes: ${ }^{122} \mathrm{Sn}$ (upper panel) and doubly magic ${ }^{132} \mathrm{Sn}$ (lower panel). The curves labeled "all" correspond to calculations that include all the calculated singleparticle states experimentally bound.

cross sections (labeled CN + DSD) as solid and dotted lines, respec tively. The DSD contribution to this cross section below $10 \mathrm{MeV}$ is small, and the experimental data [27-31] below $4 \mathrm{MeV}$ are reproduced by the compound-reaction mechanism. Above $10 \mathrm{MeV}$ the DSD mechanism becomes dominant. In the lower panel of figure 2 we have plotted the calculated $\mathrm{CN}$ capture cross section (solid line) as well as the DSD cross sections obtained with SLy4 (dashed line) and SIII (dotted line) single-particles for the ${ }^{132} \mathrm{Sn}$ target. The DSD and CN cross sections have almost the same magnitude in the $\mathrm{MeV}$ incident energy region, which may change the Maxwellian averaged cross section when the temperature of r-process is very high.

Finally we have carried out the same calculations for the deformed target nucleus ${ }^{238} \mathrm{U}$. Although coupled-channels calculations for the scattering states are expected to be more appropriate, we make the approximation of a spherical optical model and use the Koning-Delaroche potential [15]. The GDR parameters are taken from Caldwell et al. [32]. Figure 3 shows a comparison of the calculated DSD cross section obtained using SLy4 (solid line) and SIII (dashed line) deformed bound states with the experimental data $[11,24]$. The use of SLy4 bound states gives a slightly better agreement with measurements. We also calculated the DSD cross section with spherical HFBCS solutions with both Skyrme interactions (dotted and dash-dotted lines in fig. 3). The effect of the target deformation is found to be rather small. This can be explained by the fact that the sets of bound states in the spherical and deformed HFBCS solutions that contribute to the direct and semidirect amplitudes do not differ significantly in proportion to the number of bound states involved. Therefore it seems 


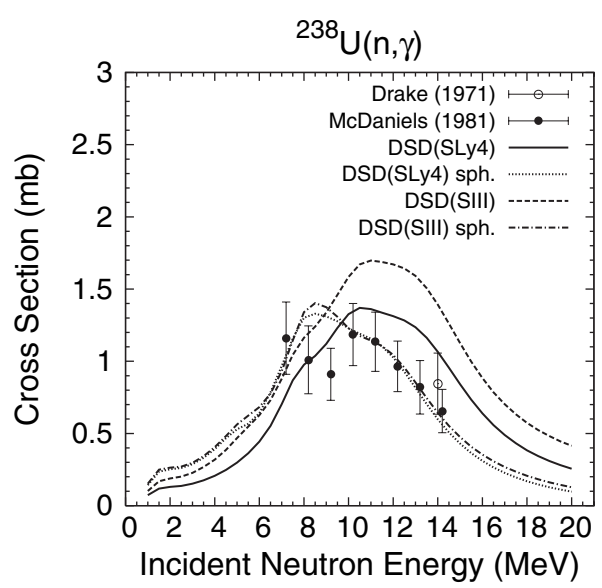

Fig. 3. Same as figure 1 for the deformed target ${ }^{238} \mathrm{U}$ with unshifted single-particle energies.

to us that the better agreement of our DSD cross section obtained using spherical bound states with experimental data from $10 \mathrm{MeV}$ to $14 \mathrm{MeV}$ is fortuitous.

\section{Conclusion}

We proposed a new technique to calculate the DSD nucleon capture cross sections. The single-particle bound states and their occupation probabilities are determined within the HFBCS model, whereas the incident wave function is calculated in the optical model. The transition amplitudes are calculated for each single-particle state using the theory of Boisson and Jang [8], with the Potokar's complex form factor for the vibration-particle coupling. The calculated occupation probabilities for ${ }^{122} \mathrm{Sn}$ agree fairly well with experimental data and the calculated neutron capture cross sections for ${ }^{208} \mathrm{~Pb}$ and ${ }^{238} \mathrm{U}$ reproduce very well the experimental cross sections with a common set of parameters for the Potokar form factor. The method was also applied to ${ }^{122} \mathrm{Sn}$ and ${ }^{132} \mathrm{Sn}$, which are important for the r-process. Whereas the compound-reaction mechanism dominates up to a few $\mathrm{MeV}$ neutron incident energies, we find that the DSD process becomes of the same magnitude for ${ }^{132} \mathrm{Sn}$.

One of us (T. W.) acknowledges the Theoretical Division at LANL for the excellent working conditions extending to him during his visit. This work has been carried out under the auspices of the National Nuclear Security Administration of the US Department of Energy at Los Alamos National Laboratory under Contract No. DE-AC5206NA25396.

\section{References}

1. R.L. Macklin, J.H. Gibbons, Rev. Mod. Phys. 37, 166 (1965).

2. R. Rauscher, F.-K. Thielemann, K.-L. Kratz, Nucl. Phys. A 621, 331c (1997).
3. R. Rauscher, F.-K. Thielemann, At. Data Nucl. Data Tables 75, 1 (2000).

4. G.E. Brown, Nucl. Phys. 57, 339 (1964).

5. C.F. Clement, A.M. Lane, J.R. Rook, Nucl. Phys. 66, 273 (1965).

6. S. Yoshida, Phys. Rev. 15, 2122 (1961).

7. B.L. Cohen, R.E. Price, Phys. Rev. 121, 1441 (1960).

8. J.P. Boisson, S. Jang, Nucl. Phys. A 189, 334 (1972).

9. H. Kitazawa, T. Hayase, N. Yamamuro, Nucl. Phys. A 307, 1 (1978).

10. B. Pålsson, J. Krumlinde, I. Bergqvist, L. Nilsson, A. Lindholm, D.C. Santry, E.D. Earle, Nucl. Phys. A 345, 221 (1980).

11. D.K. McDaniels, P. Varghese, D.M. Drake, E. Arthur, A. Lindholm, I. Bergqvist, J. Krumlinde, Nucl. Phys. A 384, 88 (1982).

12. L.C. Biendenharn, J.M. Blatt, M.E. Rose, Rev. Mod. Phys. 24, 249 (1952); J.M. Blatt, L.C. Biedenharn, Rev. Mod. Phys. 24, 258 (1952).

13. G. Longo, F. Saporetti, Nucl. Phys. A 199, 530 (1973).

14. Reference Input Parameter Library, RIPL-2, IAEA-TECDOC, International Atomic Energy Agency (2004).

15. A. Koning, J.-P. Delaroche, Nucl. Phys. A 713, 231 (2003).

16. M. Potokar, Phys. Lett. B 46, 346 (1973).

17. E. Chabanat, P. Bonche, P. Haensel, J. Meyer, R. Schaeffer, Nucl. Phys. A 635, 231 (1998).

18. T. Duguet, P. Bonche, P.-H. Heenen, Nucl. Phys. A 679, 427 (2001).

19. M. Beiner, H. Flocard, N. Van Giai, P. Quentin, Nucl. Phys. A 238, 29 (1975).

20. L. Bonneau, Phys. Rev. C 74, 014301 (2006).

21. D. Vautherin, D.M. Brink, Phys. Rev. C 5, 626 (1972).

22. D. Vautherin, Phys. Rev. C 7, 296 (1973).

23. J. Csikai, G. Peto, M. Buczko, Z. Milligy, N. Eissa, Nucl. Phys. A 95, 229 (1967).

24. D. Drake, I. Bergqvist, D.K. McDaniels, Phys. Lett. B 36, 557 (1971).

25. I. Bergqvist, D.M. Drake, D.K. McDaniels, Nucl. Phys. A 191, 641 (1972).

26. L. Bonneau. T. Kawano, T. Watanabe, S. Chiba (accepted by Phys. Rev. C).

27. V.A. Tolstikov, V.P. Koroleva, V.E. Kolesov, A.G. Dovbenko, Yu.N. Shubin, Atomnaya Energiya 24, 576 (1968).

28. M.V. Pasechnik, I.F. Barchuk, I.A. Totsky, V.I. Strizhak, A.M. Korolov, Yu.V. Hofman, G.N. Lovchikova, E.A. Koltynin, G.B. Yankov, Proc. 2nd Int. Conf. on the Peaceful Uses of Atomic Energy (United Nations, Geneva, 1958), Vol. 15, p. 18.

29. V.M. Timokhov, M.V. Bokhovko, A.G. Isakov, L.E. Kazakov, V.N. Kononov, G.N. Manturov, E.D. Poletaev, V.G. Pronyaev, Obninsk Report FEI-1921 (1988); Sov. J. Nucl. Phys. 50, 609 (1989).

30. Yu.N. Trofimov, Proc. 1st Int. Conf. on Neutr. Phys. (Kiev, 1987), Vol. 3, p. 331.

31. V.A.Tolstikov, A.N. Davletshin, E.V. Teplov, O.A. Tipunkov, Yad. Konst. 1992, 41 (1992).

32. J.T. Caldwell, E.J. Dowdy, B.L. Berman, R.A. Alvarez, P. Meyer, Phys. Rev. C 21, 1215 (1980). 